\title{
Is Minimal Invasive Plate Osteosynthesis a Viable Treatment Option for 4-part Proximal Humerus Fractures?
}

\author{
Chul-Hyun Cho \\ Department of Orthopedic Surgery, Dongsan Medical Center, Keimyung University School of Medicine, Daegu, Korea
}

Open reduction and internal fixation (ORIF) using a plate via the traditional deltopectoral approach is the gold standard for treatment of displaced proximal humerus fractures. However, it is associated with several complications including nonunion or avascular necrosis due to extensive soft tissue damage, deltoid muscle injury, and devastating infections. To overcome these problems, minimally invasive plate osteosynthesis (MIPO) for proximal humerus fractures has been introduced, and several articles report that this technique yields satisfactory radiological and clinical outcomes. ${ }^{1-3)}$

A systematic review and meta-analysis by Zhao et al. ${ }^{4)}$ reported that MIPO was superior to ORIF for the treatment of proximal humerus fractures in elderly patients. The advantage was reflected in reduced blood loss, operative time, and fracture healing time, as well as improved recovery of muscle strength. However, no significant differences were observed for rates of complications between MIPO and ORIF. Taken together, they concluded that MIPO was more suitable than ORIF for treating proximal humerus fractures in elderly patients.

Another study by Noh et al. "Minimally invasive percutaneous plate osteosynthesis via a deltoid-splitting approach with strut allograft for the treatment of displaced 3- or 4-part proximal humeral fractures" (Clin Shoulder Elbow. 2018;21(4):220-6), presented a series of 16 patients who had undergone the MIPO technique with strut allograft. All cases had complete union with mean final American Shoulder and Elbow Surgeons score of 85.4 and mean isual analogue scale score of 3.2. One patient with pseudoparalysis was converted to reverse total shoulder arthroplasty. They concluded that MIPO technique with strut allograft achieves good clinical results when applied to severely osteoporotic patients having 3- and 4-part fractures.

However, the paper by Noh et al. should be read with cau- tion, and numerous factors need to be considered. The most important point is that they did not compare outcomes and complications between 3- and 4-part fractures along with a systematic review and meta-analysis as done by Zhao et al. ${ }^{4)}$ Based on my experience, it is technically difficult to reduce and maintain the fracture fragments indirectly in real 4-part fractures as compared to 2- and 3-part fractures. Furthermore, it is also difficult to maintain the neck-shaft angle, because 4-part fractures usually occur in osteoporotic patients and are commonly combined with medial metaphyseal comminution. For these reasons, many surgeons report inferior radiological and clinical outcomes with high complication rates in 4-part fractures as compared to 2- and 3-part fractures, regardless of the surgical technique employed. Sohn and Shin ${ }^{3)}$ reported 62 cases treated by the MIPO technique, and analyzed the clinical and radiological outcomes according to fracture type. The complication rate $(72.7 \%)$ in 4-part fractures was significantly higher than other fracture types (2-part, 7.4\%; 3 part, 20.8\%). They suggest that conversion to open plating should be considered if adequate reduction is not obtained in the MIPO technique for 4-part fractures. To demonstrate the feasibility of MIPO technique in the treatment of 4-part fractures, further prospective randomized controlled studies are required.

\section{References}

1. Aguado HJ, Mingo J, Torres M, Alvarez-Ramos A, Martín-Ferrero MA. Minimally invasive polyaxial locking plate osteosynthesis for 3-4 part proximal humeral fractures: our institutional experience. Injury. 2016;47 Suppl 3:S22-8.

2. Sohn HS, Jeon YS, Lee J, Shin SJ. Clinical comparison between open plating and minimally invasive plate osteosynthesis for

\section{Correspondence to: Chul-Hyun Cho}

Department of Orthopedic Surgery, Dongsan Medical Center, Keimyung University School of Medicine, 56 Dalseong-ro, Jung-gu, Daegu 41931, Korea

Tel: +82-53-250-7729, Fax: +82-53-250-7205, E-mail: oscho5362@dsmc.or.kr, ORCID: https://orcid.org/0000-0003-0252-8741

Edirorial does not need an IRB approval.

Financial support: None. Conflict of interests: None. 
displaced proximal humeral fractures: a prospective randomized controlled trial. Injury. 2017;48(6):1175-82.

3. Sohn HS, Shin SJ. Minimally invasive plate osteosynthesis for proximal humeral fractures: clinical and radiologic outcomes according to fracture type. J Shoulder Elbow Surg. 2014;23(9):1334-40.
4. Zhao W, Zhang Y, Johansson D, Chen X, Zheng F, Li L. Comparison of minimally invasive percutaneous plate osteosynthesis and open reduction internal fixation on proximal humeral fracture in elder patients: a systematic review and metaanalysis. Biomed Res Int. 2017;2017:3431609. 